\title{
Objective Quality Assessment for Multiexposure Multifocus Image Fusion
}

\author{
Rania Hassen, Member, IEEE, Zhou Wang, Fellow, IEEE, and Magdy M. A. Salama, Fellow, IEEE
}

\begin{abstract}
There has been a growing interest in image fusion technologies, but how to objectively evaluate the quality of fused images has not been fully understood. Here, we propose a method for objective quality assessment of multiexposure multifocus image fusion based on the evaluation of three key factors of fused image quality: 1) contrast preservation; 2) sharpness; and 3) structure preservation. Subjective experiments are conducted to create an image fusion database, based on which, performance evaluation shows that the proposed fusion quality index correlates well with subjective scores, and gives a significant improvement over the existing fusion quality measures.
\end{abstract}

Index Terms - Image fusion, image quality assessment, local phase coherence, multi-focus image fusion, multi-exposure image fusion.

\section{INTRODUCTION}

$\mathbf{T}$ HE goal of image fusion is to integrate information from multiple input images to create a fused one that is more informative for human or machine perception as compared to any of the input images [1], [2]. Image fusion techniques have been used in various application areas including remote sensing, biomedical imaging, and multi-exposure multi-focus image integration. In optical remote sensing, a group of sensors may work together, each of which captures some specific aspects of spectral and/or spatial information. Fusing both the spatial and spectral information from all sensors provides a more descriptive image, and only the fused image needs to be stored for subsequent analysis of the scene [3]. In biomedical imaging, different imaging modalities are complementary in nature in acquiring different aspects of biological structures and activities. For example, magnetic resonance imaging (MRI) is often useful in revealing anatomical structures whereas metabolic activities may be captured more reliably using positron emission tomography (PET). By using fusion technologies, it is possible to obtain a single image that effectively describes anatomical structures and metabolic activities simultaneously [4]. Image fusion techniques are also

Manuscript received October 28, 2013; revised May 1, 2014 and November 6, 2014; accepted April 19, 2015. Date of publication April 29, 2015; date of current version May 19, 2015. The associate editor coordinating the review of this manuscript and approving it for publication was Dr. Sam Hasinoff.

R. Hassen is with the Department of Computer Science, Assiut University, Asyut 71515, Egypt (e-mail: rhassen@uwaterloo.ca).

Z. Wang and M. M. A. Salama are with the Department of Electrical and Computer Engineering, University of Waterloo, Waterloo, ON N2L 3G1, Canada (e-mail: zhouwang@ieee.org; m.salama@ece.uwaterloo.ca).

This paper has supplementary downloadable material available at http://ieeexplore.ieee.org., provided by the author. The material includes a real and simulated multi-focus multi-exposure images database. The total size of the file is $8 \mathrm{MB}$. Contact rhassen@mailservices.uwaterloo.ca for further questions about this work.

Color versions of one or more of the figures in this paper are available online at http://ieeexplore.ieee.org.

Digital Object Identifier 10.1109/TIP.2015.2428051 widely used in constructing high dynamic range (HDR) images by combining multiple low dynamic range (LDR) images taken with different exposures [5]. These HDR images are useful in many applications including in-vehicle cameras, surveillance in night vision, camera-guided aircraft docking, high-contrast photo development, and robot vision. Optical lenses of imaging sensors, especially those with long focal lengths, only have limited depths of field. As a result, it is impossible to have all objects with significantly different distances from the sensor to be in good focus at the same time. Therefore, another widely recognized application of image fusion is to merge multiple images of the same scene but with different focus points. Such multi-focus image fusion methods that well-preserve relevant information from the original data are highly desirable in many machine vision and image processing tasks [6], [7]. There are also applications where simultaneous multi-exposure and multi-focus image fusion are desirable. For example, in landscape astrophotography, generally night photography pushes image sensors to the limits. Recent DLSR sensors are better in handling focus and dynamic range than before. However with the physical limitations of current cameras, it is physically impossible to take a single exposure where both the stars and foreground are in focus while having minimal movement in the stars, a detailed sky, and a non-noisy foreground. Therefore, in order to create an image with a well exposed and in-focus sky and foreground, one may take multiple exposures at different focus distances and ISOs, and then blend them together by image fusion techniques to create the final result. In addition, the fact that both multi-exposure and multi-focus image fusion desires good contrast and good sharpness while preserving as much as possible the structural details motivates us to work on it as a unified problem.

Due to the large number of applications and the diversity of fusion techniques, considerable efforts have been made to develop objective performance measures for image fusion. Traditionally, the assessment of a fusion scheme is carried out by subjective evaluation, which is known to be slow, expensive, and most importantly, cannot be embedded into automated frameworks for system and parameter optimizations. A valuable alternative to subjective evaluation is objective image fusion measures that are consistent with human visual perception. It has been observed in the literature that in some special cases of multi-focus image fusion, an "ideal" fused image may be available or manually constructed, which can then be used as a ground-truth reference image to test multi-focus fusion algorithms [8]-[10]. However, obtaining an "ideal" image is not always feasible, especially in general application scenarios of multi-exposure and 
multi-focus image fusion. Therefore, objective quality assessment approaches without assuming the availability of a "ground truth" image are highly desirable. Several quality measures relate fused image quality with different aspects of information content preservation. Qu et al. [11] developed an information-theory based measure, where the summation of the mutual information (MI) between the fused and two input images is calculated to gauge the difference in quality. Xydeas and Petrovic [12] proposed an edge-based performance measure which evaluates the relative amount of edge information that is transferred from the input images to the fused image. Similarly, Wang and Liu [13] proposed a measure based on a multi-scale scheme implemented with a two-level Haar wavelet, where the edge information is retrieved from the high and band-pass components of the wavelet decomposition. A number of recent fusion performance measures build upon the structural similarity index (SSIM) [14], [15]. Piella and Heijmans [16] calculate pixel-by-pixel SSIM maps between each source image and the fused image, and then apply a weighted average method to obtain an overall evaluation of the fusion performance. In Yang et al.'s method [17], complementary or conflicting regions are distinguished from redundant regions in the two source images using SSIM maps and are treated separately. In an attempt to take into consideration human visual perception, Chen and Varshney [18] proposed an image fusion quality measure that employs the contrast sensitivity function (CSF) of the visual system and considers local spatial information on a region-by-region basis.

The purpose of the current work is to develop an objective quality model for multi-exposure multi-focus image fusion. The general approach of our method is to separate the problem into the assessment of three important factors of fused image quality - contrast preservation, sharpness, and structure preservation. Our work is partially motivated by the design principle of the SSIM approach [14], where local image fidelities are split into luminance, contrast and structural similarities. The importance of luminance preservation varies across different image fusion tasks. In the current application, we found that directly preserving the luminance of the input images is not of critical importance in improving the overall image quality, and thus we focus on contrast and structure preservations. In addition, sharpness has been recognized as an important factor in no-reference image quality assessment (IQA) [19], [20] as well as in image fusion [21]. A recently developed local phase coherence (LPC) based approach mainly makes use of local phase information in the evaluation of image sharpness [22]. This is well-suited to our framework and complements the other two factors, where local amplitude (or energy) aspect has been taken into account in contrast preservation measurement. The assessment and combination of the three critical and complementary factors lead us to a novel Fusion Quality Index (FQI). To validate the proposed approach and to compare the effectiveness and efficiency of different fusion algorithms, we carried out a subjective experiment and built a new fusion image quality database, which is probably the first of its kind. Comparisons with subjective ratings suggest that FQI
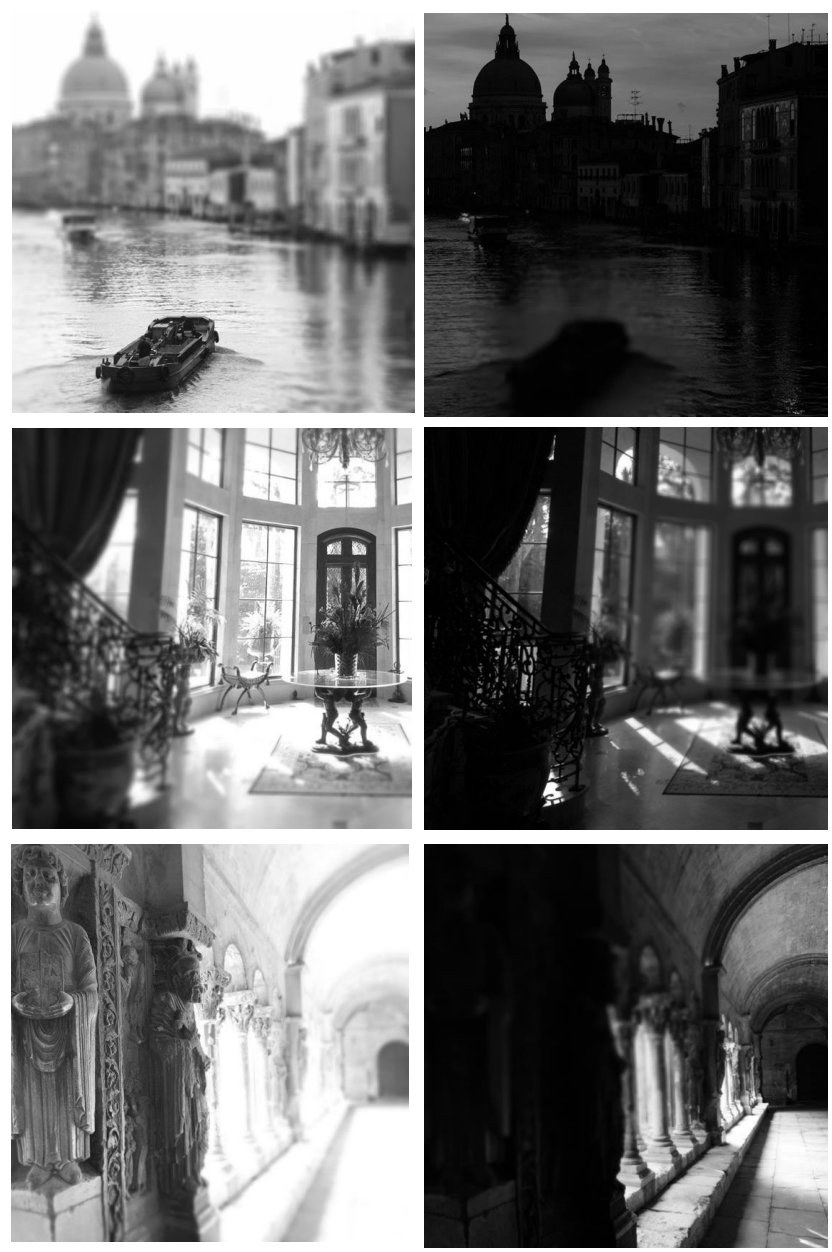

Fig. 1. Sample images with multi-exposure and multi-focus effects. Each row is a pair of images of the same scene but with different levels of exposure and different focus points.

outperforms existing fusion quality measures in the literature. To further demonstrate the potential applications of FQI, we apply it in parameter tuning of image fusion algorithms, where we show that FQI can be used as a flexible tool to guide the optimizations of image fusion algorithms.

\section{Objective Quality Assessment Method}

In photography, when a visual scene contains objects in different distances and when the camera has a limited focal length or crop factor, the camera depth-of-field is limited, causing some objects in the scene to be out-of-focus. In some other scenarios when the luminance of a scene changes dramatically across spatial locations significant information loss in the darkest and/or brightest regions, recorded as "black" (underexposed) or "white" (overexposed) values. These two common types of problem in photography can be mitigated with image fusion techniques. Examples of pairs of images with simultaneous multi-exposure and multi-focus effects are shown in Fig. 1. Perception studies indicate that the best fusion strategy is task dependent [23] and that humans exploit different features in an image for different tasks [24]. Therefore, an ideal fusion quality measure should be task specific, and the "best" fusion algorithm changes from task to task. In case of multi-exposure and multi-focus fusion, the 
task is to integrate input images with different focal points and/or different exposures to generate one fused image with sharper features and clearer structural details as compared to the source images. Assume we have $L$ input images $\left\{A_{l}\right\}=\left\{A_{1}, A_{2}, A_{3}, \ldots . ., A_{L}\right\}$ which are fused to produce image $F$. We found that three characteristics - contrast preservation, sharpness, and structure preservation - are critically important in accounting for the visual quality of $F$ given $\left\{A_{l}\right\}$.

\section{A. Contrast Preservation Assessment}

There are several variations of contrast definitions in the vision science literature. The general idea is to normalize the foreground intensity signal strength with the background luminance. This approach has some limitations when applied in complex natural images viewed in practical application environment. Specifically, compared with the ideal darkroom environment in standard vision science experiments, the background luminance used as normalization factor is not well-controlled in case of practical application environment. A simple method is to integrate the background luminance in the entire environment as the normalization factor for contrast computation. This leads to applying the same normalization factor to everywhere in the image. As such, it was found in the literature of image quality assessment that using only the standard deviation without a normalization factor leads to a simple, useful, and robust local contrast measure [14]. Following the same approach in [14], we measure contrast preservation locally using a sliding window approach, which results in a map that indicates the spatial variation of local contrast preservation. The complication is that in the case of multiple input images, multiple input image patches are available at each spatial location, which may have different contrasts. A useful assumption is that the input image patch that has the highest contrast is the most informative, and the contrast of the fused image patch should be close to the contrast of the most informative image patch. Figure 2 illustrates the process of the proposed contrast preservation assessment method together with image examples for the case of two input images. Let $a_{l, k}$ and $f_{k}$ be the $k$-th local image patches of the $l$-th input and fused images, respectively. The local contrast similarity assessment function is defined as

$$
c\left(\left\{a_{l, k}\right\} ; f_{k}\right)=\frac{2 \sigma_{f_{k}} \max _{l}\left\{\sigma_{a_{l, k}}\right\}+C_{1}}{\sigma_{f_{k}}^{2}+\left(\max _{l}\left\{\sigma_{a_{l, k}}\right\}\right)^{2}+C_{1}},
$$

where $\sigma_{a_{l, k}}$, and $\sigma_{f_{k}}$ are the standard deviations of local image patches in the $l$-th input and fused images, respectively, $\max _{l}\left\{\sigma_{a_{l, k}}\right\}$ is the maximum standard deviation of all $\sigma_{a_{l, k}}$ for $l \stackrel{l}{=} 1,2, \ldots, L$ and $C_{1}$ is a positive stabilizing constant. This local measure is applied using a sliding window that runs across the image space, leading to a contrast preservation map bounded between 0 and 1 , where a higher value corresponds to better contrast preservation. Direct averaging is then applied to pool the map into a single contrast preservation measure:

$$
Q_{c}\left(F \mid\left\{A_{l}\right\}\right)=\frac{1}{N} \sum_{k=1}^{N} c\left(\left\{a_{l, k}\right\} ; f_{k}\right),
$$

where $N$ is the total number of patches.

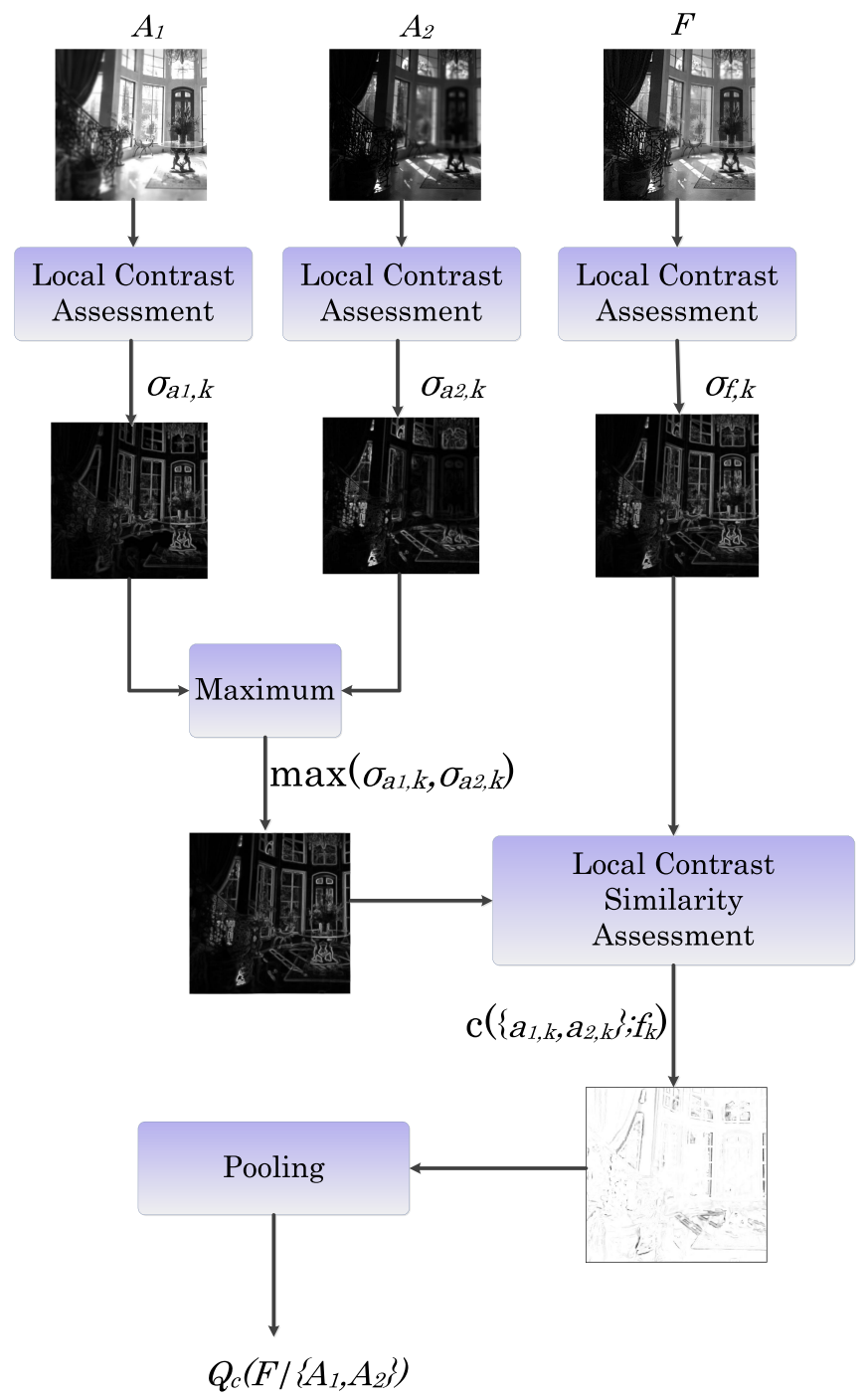

Fig. 2. Framework of contrast preservation assessment with examples for the case of two input images.

\section{B. Sharpness Assessment}

Sharpness is an important determinant in visual perception of image quality [22]. In our earlier work, an LPC-based sharpness index (LPC-SI) was proposed [22], which is a NR approach and achieved highly competitive performance when tested using publicly available image databases. In addition, LPC-SI produces a dense sharpness map that indicates the spatial variations of perceptual sharpness, a feature that is lacking in other sharpness measures in the literature [22].

Here we apply the LPC-SI approach to the fused image, which is first passed through a series of $N$-scale $M$-orientation complex log-Gabor filters without any subsequent down-sampling process. Let $c_{i j k}$ be the resulting complex coefficient at the $i$-th scale, the $j$-th orientation, and the $k$-th spatial location. The LPC-based sharpness measure at the $k$-th location is given by

$$
H_{k}=\frac{\sum_{j=1}^{M}\left|c_{1 j k}\right| \cos \left(\Phi\left\{\prod_{i=1}^{N} c_{i j k}^{w_{i}}\right\}\right)}{\sum_{j=1}^{M}\left|c_{1 j k}\right|+C_{2}},
$$




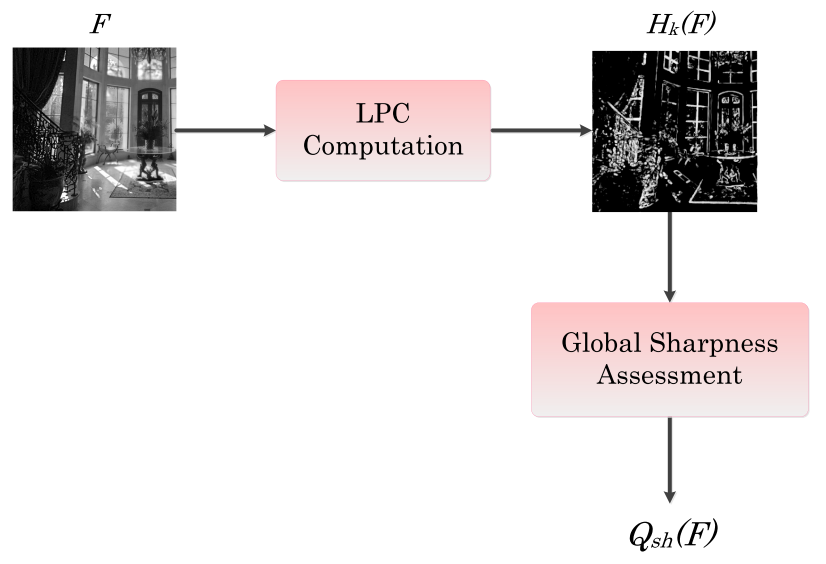

Fig. 3. Framework of sharpness assessment with examples.

where $\Phi\{\cdot\}$ denotes the angle of a complex number, $w_{i}$ are an optimal set of factors to relate the phases across different scales, $\cos \left(\Phi\left\{\prod_{i=1}^{N} c_{i j k}^{w_{i}}\right\}\right)$ measures the phase coherence at the $j$-th orientation and the $k$-th spatial location, and $C_{2}$ is a constant to avoid instability in case of small magnitude coefficients. The magnitudes of the finest scale coefficients $c_{1 j k}$ across different orientations $j$ are used as weighting factor, where higher magnitude leads to higher weight.

The collection of LPC measures computed for the fused image $H_{k}(F)$ at all spatial locations constitutes a sharpness map of the fused image. The overall image sharpness is obtained by pooling the LPC map using a weighted averaging of ranked LPC values $H_{(k)}(F)$ as follows

$$
Q_{s h}(F)=\frac{\sum_{k=1}^{K} u_{k} H_{(k)}(F)}{\sum_{k=1}^{K} u_{k}},
$$

where $u_{k}$ is the weight assigned to the $k$-th ranked LPC value $H_{(k)}(F)$ and is computed as an exponentially decaying function given by [22]

$$
u_{k}=\exp \left[-\left(\frac{k-1}{K-1}\right) / \beta_{k}\right]
$$

which gives a weight 1 to the highest LPC value and the decaying speed is controlled by the parameter $\beta_{k}$. As in [22], the parameters are set as $M=8, N=3, s_{1}=1, s_{2}=3 / 2$, $s_{3}=2, w_{1}=1, w_{2}=-3, w_{3}=2, \beta_{k}=1 e-4$, and $C_{2}=2$, respectively. These parameters are set empirically, but are found to be insensitive to the overall performance. Figure 3 depicts the framework of the sharpness assessment procedure with an image example.

\section{Structure Preservation Assessment}

The SSIM method [14] provides a useful design principle as well as a simple and effective method to measure the structural fidelity between images. Following the SSIM approach, we evaluate structural fidelity locally using a sliding window approach, which leads to a structure preservation map. However, since multiple input images are available in image fusion applications, multiple structure preservation maps are created, each computed by comparing one input image with the fused image. Novel strategies need to be developed to pool these maps into a scalar evaluation regarding the overall structure preservation.

Figure 4 shows the diagram of the proposed structure preservation assessment algorithm along with image examples for the case of two input images. Let $a_{l, k}$, and $f_{k}$ be the $k$-th local image patches in the $l$-th input and fused images, respectively, the structural fidelity evaluation is conducted between each of the input image patches and their fusion result:

$$
S\left(a_{l, k}, f_{k}\right)=\frac{\sigma_{a_{l, k} f_{k}}+C_{3}}{\sigma_{a_{l, k}} \sigma_{f_{k}}+C_{3}}
$$

where $\sigma_{a_{l, k}}$ and $\sigma_{f_{k}}$ are the local standard deviations of the local image patches, $\sigma_{a_{l, k} f_{k}}$ is the cross correlation between the two corresponding patches, respectively, and $C_{3}$ is a stabilizing constant (as in [14]). The collections of $S\left(a_{l, k}, f_{k}\right)$ values at all spatial locations constitute $L$ structure preservation maps that deliver information about how image structures are preserved in the fused image. Multi-focus input images contain complementary sharp regions, where the regions with sharper content tend to be better focused and thus better preserve local image structures. It is thus reasonable to give more importance to the sharper regions. As illustrated in Fig. 4, we use LPC-based sharpness measure to create sharpness maps, which are subsequently used to weigh the structure preservation maps computed from both input images. The overall structure preservation measure is given by

$$
Q_{s}\left(F \mid\left\{A_{l}\right\}\right)=\frac{1}{L} \sum_{l=1}^{L} \frac{\sum_{k=1}^{K} H_{k}\left(A_{l}\right) S\left(a_{l, k}, f_{k}\right)}{\sum_{k=1}^{K} H_{k}\left(A_{l}\right)}
$$

where $H_{k}\left(A_{l}\right)$ is the LPC map of the $l$-th input image $A_{l}$. Since the local structure preservation measure (6) is upper-bounded by 1 , the value of $Q_{s}\left(F \mid\left\{A_{l}\right\}\right)$ is also upper-bounded by 1 .

\section{Overall Quality Assessment}

The contrast preservation, sharpness, and structure preservation measures descried above address different aspects about fused image quality and are complementary to each other. By combining these measures, we obtain an overall image fusion quality index (FQI) given by

$$
Q\left(F \mid\left\{A_{l}\right\}\right)=Q_{c}\left(F \mid\left\{A_{l}\right\}\right) \cdot Q_{s h}(F) \cdot Q_{s}\left(F \mid\left\{A_{l}\right\}\right) .
$$

Since all three components are upper-bounded by 1 , the overall FQI measure is also upper-bounded by 1 , which corresponds to perfect contrast preservation, sharpness and structure preservation simultaneously.

\section{SUBJeCTIVE EXPERIMENT}

Because the human observers are the ultimate consumers in most applications, subjective user study is considered as the most reliable approach to evaluate the quality of fused images. The fusion community has captured and shared a number of multi-focus and multi-exposure reference images for algorithm development and assessment. A variety of methods have been used for subjective evaluation of the quality of fused images. 


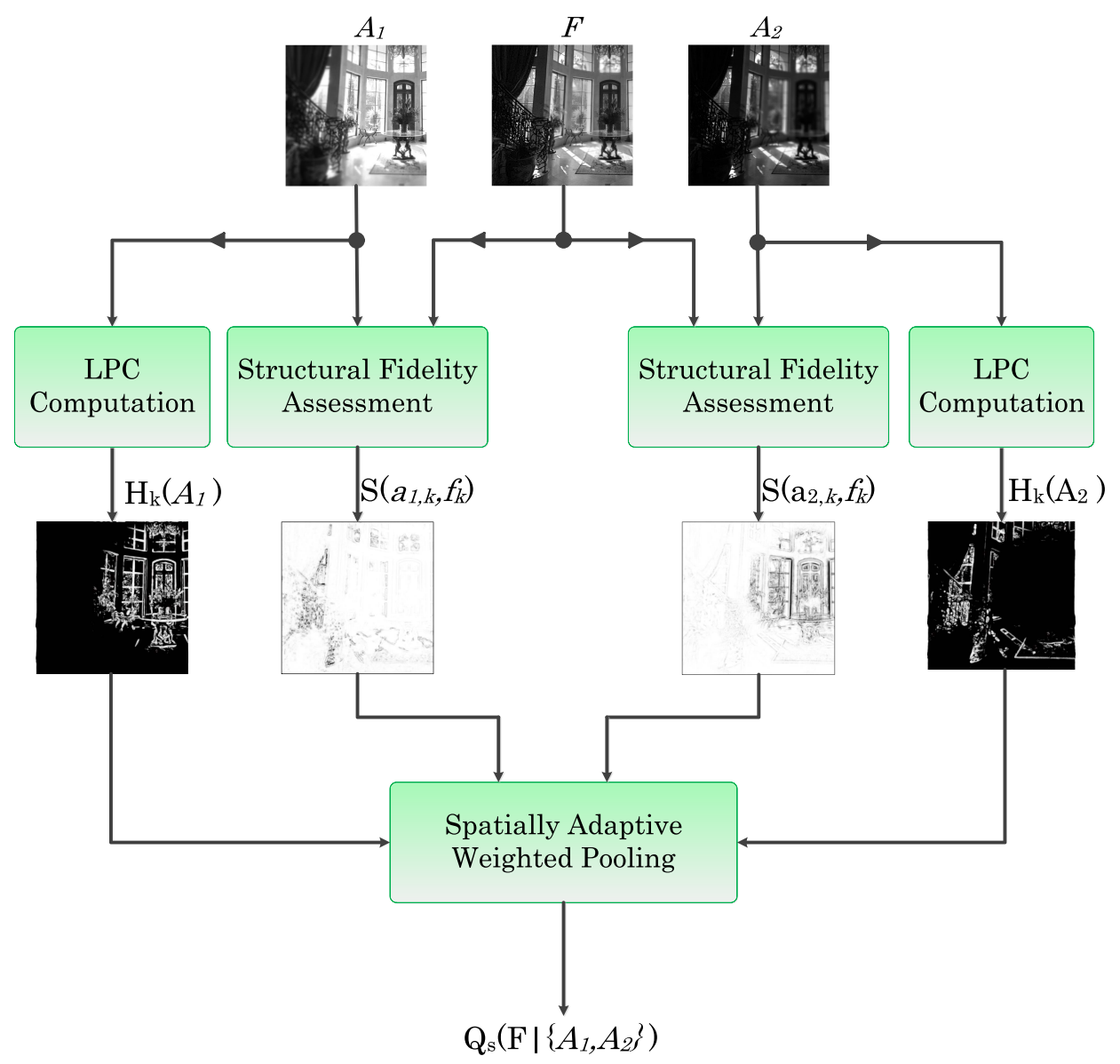

Fig. 4. Framework of structure preservation assessment with examples for the case of two input images.

Typically, a subject is asked to rank or rate the quality of the image on a linear or ordinal scale. Three approaches are discussed in the literature [25] - simple ranking, Single Stimulus Continuous Quality Evaluation (SSCQE), and Double Stimulus Continuous Quality Evaluation (DSCQE). Single and double stimulus methods represent categorical rating, in which observers judge the quality of a single and a pair of images on a fixed 5-point scale. In [25], a paired comparison was used to evaluate the relative advantage of one or neither of the two offered fusion results as perceived by the test audience. The method is straightforward and popular in computer graphics [26], but is tedious if a large number of conditions needs to be compared. Another type of comparative evaluation used in the literature (and is adopted in the current work) is to rank a set of images from the best to the worst, such as the work in [27] and [28], which follows the standard CCIR procedure [29].

To the best of our knowledge, currently there is no publiclyavailable subject-rated image fusion database that can be directly employed to test and compare algorithms developed for image fusion quality assessment. Therefore, we build a database of simultaneous multi-exposure and multi-focus images. The database is created from 14 pairs of source images of $560 \times 560$ or $480 \times 480$ resolutions, one overexposed and the other underexposed. Each pair of images is further altered by simulated out-of-focus at complementary spatial locations, where the out-of-focus effect is simulated using foveated filters with different fixation points [30]. Examples of the resulting multi-exposure multi-focus image pairs are given in Fig. 1.

Six fusion algorithms with various rules to extract and combine image structures from the input image pairs are employed to create the fused images. Many fusion algorithms involve multi-resolution decompositions followed by fusion rules that impose more emphasis on the input image that has more salient features in a spatially adaptive manner, where typical fusion rules include choose maximum absolute coefficient (CM), weighted average (WA), and choose maximum with consistency verification (CMCV). Table I summarizes the decomposition methods and the fusion rules employed by the six fusion algorithms, which include (1) pixel average (AVG), (2) Laplacian pyramid decomposition followed by CM fusion (LAP-CM), (3) contrast pyramid decomposition followed by $\mathrm{CMCV}$ fusion (COP-CMCV), (4) ratio pyramid decomposition followed by WA fusion (RAP-WA), (5) discrete wavelet transform decomposition followed by CM fusion (DWT-CM), and (6) generalized random walk based probabilistic weighted fusion (GRW-PW) [31]. The implementation of Algorithm (1) is straightforward and that of Algorithm (6) was provided by the authors of [31] using author recommended parameters $\overline{\sigma_{w}}=0.1, \gamma=1.0$, and $\eta=4$. The rest of the algorithms all follow a multiresolution framework, and our implementations were based 
TABLE I

Fusion Rules AND DeCOMPositions IN THE IMAGE Fusion Algorithms

\begin{tabular}{|c|l|l|}
\hline Fusion Algorithm & Decomposition & Fusion Rule \\
\hline AVG & None & Average pixel intensities \\
LAP-CM & 2-level Laplacian pyramid & Choose Max of absolute coefficients in details sub-bands \\
COP-CMCV & 2-level contrast pyramid & Choose Max of absolute coefficients in details sub-bands with consistency verification \\
RAP-WA & 2-level ratio pyramid & Weighted average of absolute coefficients in details sub-bands \\
DWT-CM & 2-level discrete wavelet transform & Choose Max of absolute coefficients in details sub-bands \\
GRW-PW & None & Generalized random walk based probabilistic weighting \\
\hline
\end{tabular}

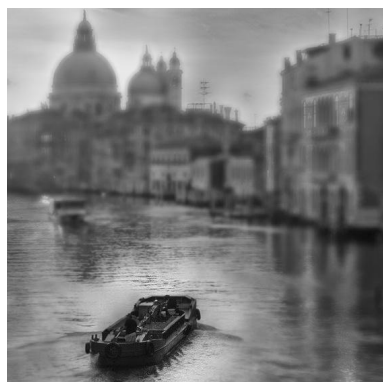

$\mathrm{FQI}=0.4868$

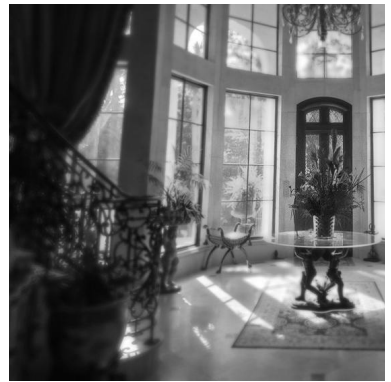

$\mathrm{FQI}=0.6269$

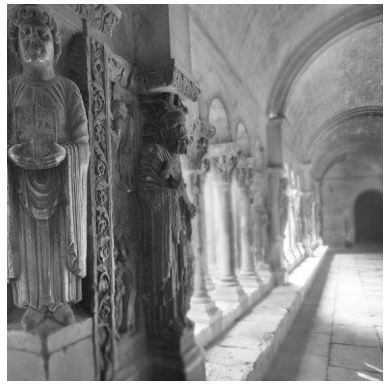

$\mathrm{FQI}=0.6114$

(a)

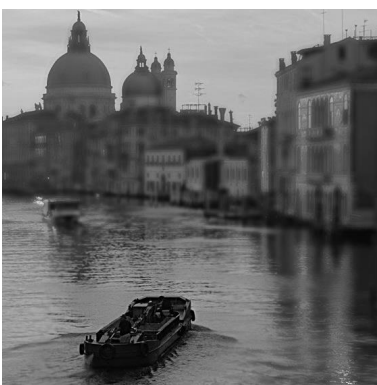

$\mathrm{FQI}=0.6569$

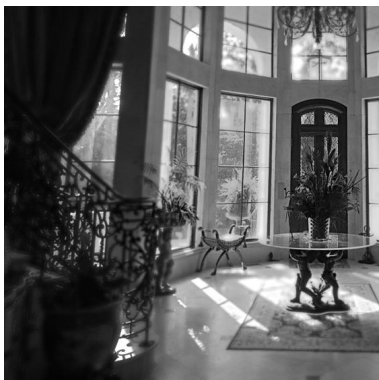

$\mathrm{FQI}=0.7467$

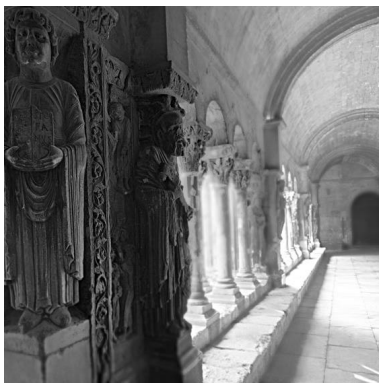

$\mathrm{FQI}=0.6844$

(b)

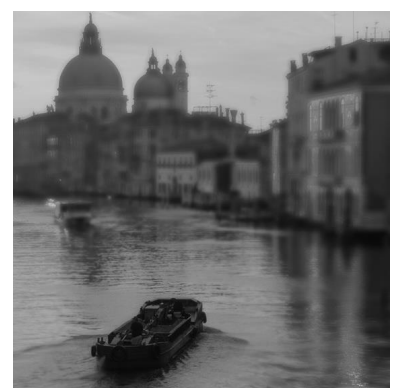

$\mathrm{FQI}=0.5271$

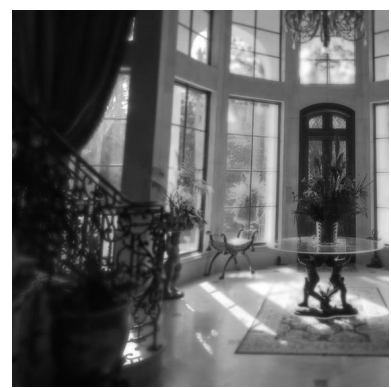

$\mathrm{FQI}=0.6219$

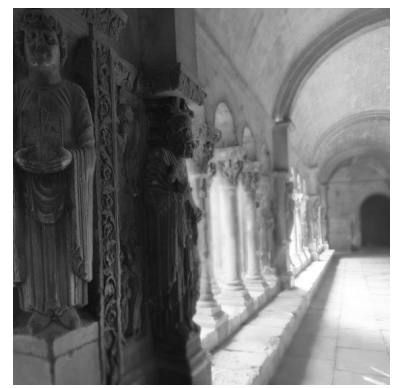

$\mathrm{FQI}=0.5321$

(c)

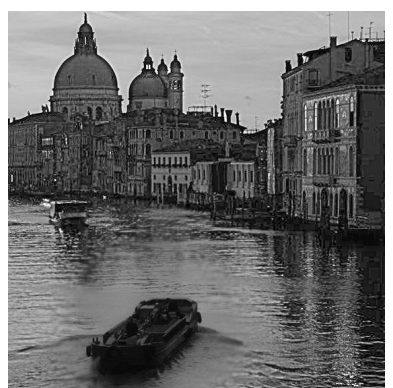

$\mathrm{FQI}=0.5309$

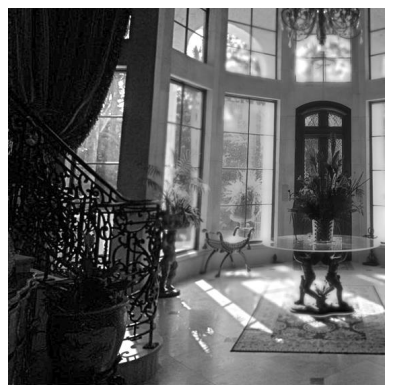

$\mathrm{FQI}=0.6643$

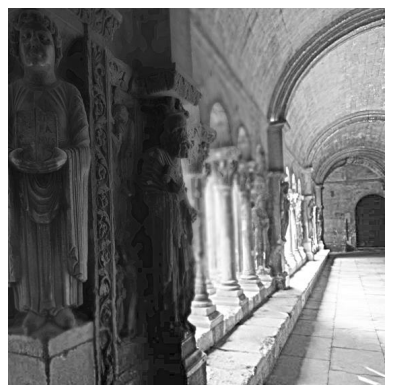

$\mathrm{FQI}=0.6045$

(d)

Fig. 5. Fused images and their corresponding FQI values for the input image pairs shown in Fig. 1 using (a) GRW-PW, (b) LAP-CM, (c) AVG, and (d) RAP-WA algorithms.

on Rockingers' MATLAB toolbox [32]. Figure 5 shows examples of the fusion results for the input images in Fig. 1, together with their FQI values.

We conducted a subjective test using the multi-exposure multi-focus image fusion database described above. Twenty naive subjects, including 11 males and 9 females, aged between 19 and 30, participated in the study. A 30-inch LCD monitor with $2560 \times 1600$ resolution was employed, which allowed us to display on the same screen a full set of 6 fused images created by the aforementioned fusion algorithms, together with the corresponding pair of multi-exposure multi-focus input images. The positions of the fused images were placed in random order to avoid any positional selection bias. The test environment had no reflecting ceiling walls or floors and was kept constant during the test. Before the test, a brief instruction session was performed where the subjects were shown sample images and the experiment process was explained. For each set of test images, the subjects were asked to give a ranking score between 1 and 6 to each fused image on the screen, where " 1 " stands for the best perceptual quality, and "6" denotes the worst quality. The subject performed the tests individually with no time limit. After the subjective test, a statistical analysis was performed and two subjects are identified as outliers and their corresponding scores were removed. The subjective rankings for the remaining 18 subjects for each image is averaged, resulting in its mean ranking score within the set. The ranking scores were 
TABLE II

KRCC ANd SRCC Performance of AVerage SubJeCt

\begin{tabular}{|c|cc|cc|}
\hline \multirow{2}{*}{$\begin{array}{c}\text { Image } \\
\text { Set }\end{array}$} & \multicolumn{2}{|c|}{ KRCC } & \multicolumn{2}{c|}{ SRCC } \\
\cline { 2 - 5 } & $\mu$ (subject) & $\sigma$ (subject) & $\mu$ (subject) & $\sigma$ (subject) \\
\hline BelguimHouse & 0.7961 & 0.2455 & 0.8689 & 0.2107 \\
Boat & 0.8039 & 0.1499 & 0.8789 & 0.1276 \\
Cafa & 0.7098 & 0.2460 & 0.7882 & 0.2422 \\
Church & 0.8509 & 0.1993 & 0.9126 & 0.1486 \\
Foyer & 0.8482 & 0.1301 & 0.9216 & 0.0837 \\
Garden & 0.8051 & 0.1103 & 0.8908 & 0.0880 \\
House & 0.8849 & 0.0983 & 0.9396 & 0.0632 \\
Lamp & 0.7302 & 0.1992 & 0.8289 & 0.1729 \\
MAC & 0.8039 & 0.1771 & 0.8789 & 0.1383 \\
Office & 0.7991 & 0.1849 & 0.8703 & 0.1538 \\
Parking & 0.8353 & 0.1108 & 0.9126 & 0.0759 \\
Radio & 0.6941 & 0.2615 & 0.7882 & 0.2328 \\
Square & 0.7725 & 0.1811 & 0.8622 & 0.1579 \\
Statue & 0.7647 & 0.1455 & 0.8521 & 0.1144 \\
\hline Average & 0.7928 & 0.1743 & 0.8710 & 0.1436 \\
\hline
\end{tabular}

also converted into linear quality scores using Thurstone's statistical model [33], [34] based on maximum-aposterior (MAP) estimation. This was done by first computing a winning frequency matrix $C$ from all possible paired comparisons for all fusion results. The probability $P(A>B)$ is then estimated by using the empirical proportion of people preferring option $A$ over option $B$, given as $C_{A, B}=\left(C_{A, B}+C_{B, A}\right)$. Finally, the linear quality scores are estimated by maximizing the inverse of cumulative density function of the Gaussian random quality difference $A-B$. The converted linear quality scores preserve the mean ranking scores in each image set and are employed as the mean opinion scores (MOSs) in the subsequent evaluations.

Three criteria are employed to evaluate the level of agreement between the subjective opinions and the predictions by quality assessment models. These include Kendall's rankorder correlation coefficient (KRCC), Spearman's rank-order correlation coefficient (SRCC), and Pearson's linear correlation coefficient (PLCC) between the subjective scores and the quality measures. All three criteria range between -1 to 1 , which represent perfect disagreement or agreement between model prediction and subjective opinion, respectively.

To provide a reference point in evaluating the performance of FQI, we evaluate how an average subject would behave in such a test. To do this, for each image set, we compute the KRCC and SRCC values between the MOSs and the ranking scores given by each of the individual subjects. When this is done for all 18 subjects, we compute the mean $(\mu)$ and standard deviation $(\sigma)$ of the KRCC and SRCC values over all subjects. The average subject performance is summarized in Table II. Generally, an average subject's opinions are well correlated with the mean opinions of all subjects. Meanwhile, there still exists substantial variations between different subjects, reflected by the significant std values in Table II. These results give a useful baseline to evaluate how an objective quality prediction model behaves relative to a typical human subject.

\section{Performance Evaluation and Comparison}

To validate the proposed FQI measure, we first examine how each of its three components, $Q_{c}, Q_{s h}$ and $Q_{s}$, contributes to the quality prediction performance. Table III shows the KRCC, SRCC and PLCC performances of each component, along with the performance of $\mathrm{FQI}$ that combines all three components. These include the results for each image set as well as the average result over all image sets. It can be seen that all three components provide significant positive correlations with subjective opinions, while the overall FQI delivers substantial and consistent performance improve over all three components. Comparing Table III with Table II, we also observe that FQI often outperforms an average subject, though the improvements are typically within 1 std of the variations between human subjects.

To further evaluate the performance of the proposed FQI method, we compare it against seven state-of-the-art image fusion quality measures (where parameters in all these measures are set as their default values). These measures include: (1) normalized mutual information (NMI) [11], which quantifies the amount of information transferred from the source images to the fused images by summing the mutual information between the fused and two input images; (2) edge dependent weighted SSIM (EW-SSIM) [16], where SSIM [14] between the fused and two input images are computed, followed by edge dependent weighting; (3) edge information preservation (EIP) [12], which measures the amount of edge information in terms of edge strength and orientation that is transferred from the input images to the fused image; (4) selective weighted SSIM (SW-SSIM) [17], which not only compute SSIM measures between the input and fused images, but also the SSIM between the input images. These are followed by adaptively selecting between local averaging and taking the maximum of local SSIM values; (5) edge strength (ES) [18], which applies human visual contrast sensitivity function (CSF) in the frequency domain and then create an edge strength maps for the input and fused images, followed by salience weighted pooling; (6) multiscale edge preservation (MEP) [13], which decomposes the input and fused images using a two-level Haar wavelet transform and estimates edge preservation in horizontal, vertical and diagonal directions at each scale, followed by energy weighted pooling across space and scale; (7) saliency weighted contrast preservation (SWCP) [27], which filters the input and fused images by a CSF and computes local contrast maps, followed by contrast preservation evaluation and saliency weighted pooling.

Table IV shows the KRCC, SRCC and PLCC analysis results of all 14 image sets for all eight quality prediction models (including the 7 existing models described above and FQI). The recommended algorithm parameters for the 7 fusion assessment measures were used to obtain the results of comparison. The parameters used in fusion measures are given in Table V. The average performance over all image sets are also given. Among the existing models, EW-SSIM [16] and EIP [12] produce quite competitive results, while the proposed FQI measure results in the best performance in all three criteria.

To better understand the relationships between the fusion quality measures under study, a visual representation of the similarities between the fusion quality models is created using 
TABLE III

KRCC, SRCC AND PLCC PERFoRMANCE EVALUATION OF FQI AND ITS ThREE COMPONENTS

\begin{tabular}{|c|c|c|c|c|c|c|c|c|c|c|c|c|}
\hline \multirow{2}{*}{$\begin{array}{c}\text { Image } \\
\text { Set }\end{array}$} & \multicolumn{4}{|c|}{ KRCC } & \multicolumn{4}{|c|}{ SRCC } & \multicolumn{4}{|c|}{ PLCC } \\
\hline & $Q_{c}$ & $Q_{s h}$ & $Q_{s}$ & FQI & $Q_{c}$ & $Q_{s h}$ & $Q_{s}$ & FQI & $Q_{c}$ & $Q_{s h}$ & $Q_{s}$ & FQI \\
\hline BelguimHouse & 0.7333 & 0.6000 & 0.8667 & 0.8667 & 0.8286 & 0.6571 & 0.9429 & 0.9429 & 0.9682 & 0.8469 & 0.7864 & 0.9533 \\
\hline Boat & 0.6000 & 1.0000 & 0.0667 & 0.8667 & 0.7714 & 1.0000 & 0.0286 & 0.9429 & 0.0858 & 0.9596 & 0.8455 & 0.9444 \\
\hline Cafa & 0.8667 & 0.3333 & 0.6000 & 0.8667 & 0.9429 & 0.4286 & 0.7714 & 0.9429 & 0.6623 & 0.7847 & 0.8794 & 0.9621 \\
\hline Church & 0.7333 & 0.7333 & 0.4667 & 0.8667 & 0.8286 & 0.8857 & 0.6000 & 0.9429 & 0.7505 & 0.9490 & 0.8547 & 0.9276 \\
\hline Foyer & 0.7333 & 0.8667 & 0.6000 & 0.8667 & 0.8857 & 0.9429 & 0.6571 & 0.9429 & 0.7148 & 0.9704 & 0.8465 & 0.9341 \\
\hline Garden & 0.6901 & 0.6901 & 0.6901 & 0.9661 & 0.8117 & 0.8407 & 0.8117 & 0.9856 & 0.8521 & 0.9167 & 0.8542 & 0.9460 \\
\hline House & 0.6901 & 0.9661 & 0.5521 & 0.8281 & 0.7827 & 0.9856 & 0.6377 & 0.9276 & 0.8239 & 0.9622 & 0.8301 & 0.9611 \\
\hline Lamp & 0.4667 & 0.7333 & 0.2000 & 0.8667 & 0.5429 & 0.8857 & 0.3143 & 0.9429 & 0.3907 & 0.9246 & 0.8622 & 0.9820 \\
\hline MAC & 0.7333 & 0.3333 & 0.7333 & 0.8667 & 0.8857 & 0.3714 & 0.8286 & 0.9429 & 0.6366 & 0.7733 & 0.9235 & 0.9243 \\
\hline Office & 0.7333 & 0.6000 & 0.8667 & 0.7333 & 0.8857 & 0.7714 & 0.9429 & 0.8286 & 0.8706 & 0.9153 & 0.9237 & 0.9576 \\
\hline Parking & 0.7333 & 1.0000 & 0.7333 & 1.0000 & 0.8286 & 1.0000 & 0.8857 & 1.0000 & 0.8987 & 0.9809 & 0.8989 & 0.9765 \\
\hline Radio & 0.7333 & 0.8667 & 0.7333 & 1.0000 & 0.8286 & 0.9429 & 0.8286 & 1.0000 & 0.9165 & 0.8959 & 0.8868 & 0.9611 \\
\hline Square & 0.7333 & 0.6000 & 0.7333 & 1.0000 & 0.8286 & 0.6571 & 0.8286 & 1.0000 & 0.7678 & 0.7977 & 0.9533 & 0.9821 \\
\hline Statue & 0.6000 & 1.0000 & 0.7333 & 0.8667 & 0.6571 & 1.0000 & 0.8286 & 0.9429 & 0.9510 & 0.9705 & 0.8997 & 0.9894 \\
\hline Average & 0.6986 & 0.7373 & 0.6125 & 0.8901 & 0.8078 & 0.8121 & 0.7076 & 0.9489 & 0.7350 & 0.9034 & 0.8746 & 0.9573 \\
\hline
\end{tabular}

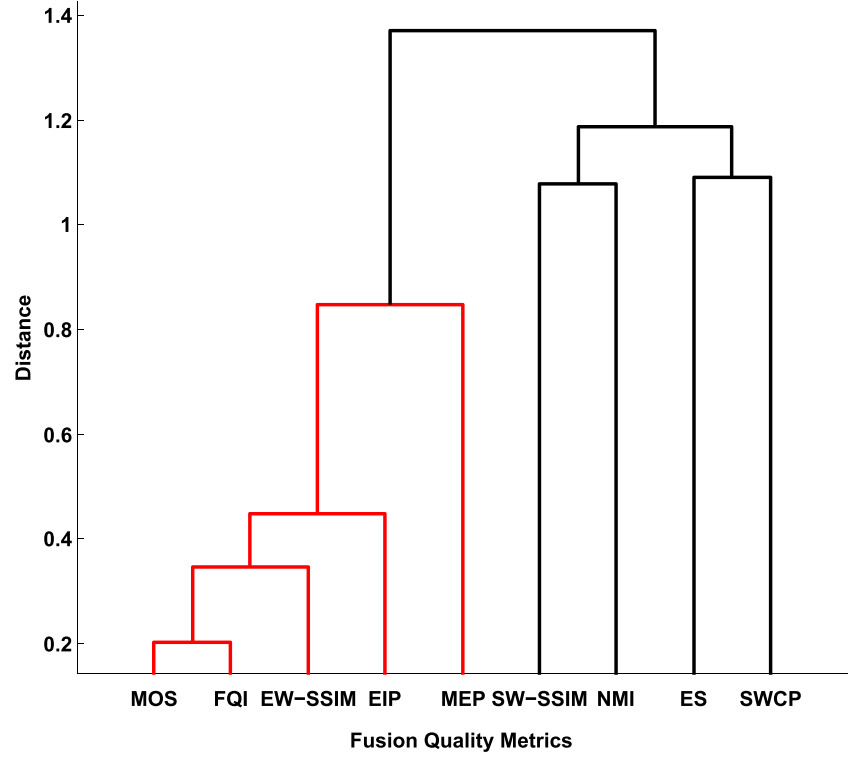

Fig. 6. Similarity dendrogram of MOS and objective image fusion quality measures.

a dendrogram plot shown in Fig. 6. Specifically, KRCCs between the eight fusion quality models are first computed and averaged across image sets. The dendrogram then transforms the KRCC values into distances and clusters them using the unweighted pair group method with arithmetic mean (UPGMA) algorithm [35], [36], where Euclidean distance is used as a distance metric for data clustering. In Fig. 6, the horizontal axis represents the fusion quality models (along with MOS), which are referred to as the leaf nodes. The vertical axis is labeled by distances between the nodes. The height of the node can be thought of as the distance between the right and left sub-branch clusters. As in earlier correlation studies (Table IV), this dendrogram suggests that EW-SSIM and EIP are the most competitive measures in previous algorithms, and the proposed FQI measure outperforms all existing algorithms and is the closest to MOS.

We also carried out a statistical significance analysis using the approach introduced in [37]. The goal of the analysis is to look at the quality prediction residuals between MOS values and the predictions from two competing objective quality assessment models, and then perform a hypothesis testing on whether the residuals are coming from the same distribution and are statistically indistinguishable. Table VI summarizes the results by comparing every pair of objective image fusion quality assessment models mentioned above, where a symbol "-_" denotes that the two models are statistically indistinguishable, " 1 " denotes the model of the row is statistically better than that of the column, and " 0 " denotes that the model of the column is better than that of the row. The results generally agree with the dendrogram in Fig. 6. FQI appears to be statistically better than almost all other models, except for EW-SSIM. This might be due to the underlying assumptions made by this analysis approach, where the quality prediction residuals need to be Gaussian distributed, an assumption that are not strictly satisfied by the quality assessment models being tested.

To verify the proposed FQI measure, we test it on multi-exposure multi-focus images captured in real scenes. A set of 8 images were acquired using Nikon D5200 with AF-S DX NIKKOR 35mm f/1.8G lens. Two images were captured for the same scene, with different near far focus points and with different exposure levels to show all foreground and background details. Sample images are shown in Fig. 7, where the images in the first row have low exposure value and far focus point to show details outside of the windows, while the complementary images in the second row have near focus point on the indoor objects and high exposure level. The same six fusion algorithms were used to fuse the pair of real multi-exposure multi-focus images. A new subjective test was carried out using the new fusion results. 19 subjects were asked to rank the images using the same procedure described before. One outlier was excluded and the mean opinion scores are computed after removing the outlier. The SRCC performance of the 8 fusion assessment measures evaluated using the subjective data on real images is summarized in Table VII. Again, the proposed FQI method delivers the most competitive performance among all fusion quality assessment measures. On the other hand, the SRCC values are significantly lower than the simulated fusion test case (as in Table IV). This may 
TABLE IV

KRCC, SRCC ANd PLCC Performance EVAluation of Image Fusion Quality Models

\begin{tabular}{|c|c|c|c|c|c|c|c|c|}
\hline & \multicolumn{8}{|c|}{ Kendall Rank-Order Correlation Coefficient (KRCC) } \\
\hline Image Set & NMI [11] & EW-SSIM [16] & EIP [12] & SW-SSIM [17] & ES [18] & MEP [13] & SWCP [27] & FQI \\
\hline BelguimHouse & 0.0667 & 0.7333 & 1.0000 & 0.6000 & 0.0667 & 0.8667 & 0.2000 & 0.8667 \\
\hline Boat & 0.0667 & 0.4667 & 0.4667 & 0.2000 & 0.0667 & 0.3333 & 0.6000 & 0.8667 \\
\hline Cafa & 0.2000 & 0.8667 & 0.7333 & 0.0667 & 0.3333 & 0.6000 & 0.0667 & 0.8667 \\
\hline Church & 0.0667 & 0.7333 & 0.6000 & 0.0667 & 0.6000 & 0.4667 & 0.2000 & 0.8667 \\
\hline Foyer & 0.0667 & 0.8667 & 0.6000 & 0.0667 & 0.6000 & 0.6000 & 0.4667 & 0.8667 \\
\hline Garden & 0.2760 & 0.8281 & 0.6901 & 0.1380 & 0.9661 & 0.2760 & 0.4140 & 0.9661 \\
\hline House & 0.0000 & 0.6901 & 0.5521 & 0.1380 & 0.1380 & 0.4140 & 0.4140 & 0.8281 \\
\hline Lamp & 0.0667 & 0.6000 & 0.6000 & 0.0667 & 0.0667 & 0.6000 & 0.0667 & 0.8667 \\
\hline MAC & 0.0667 & 0.2000 & 0.7333 & 0.0667 & 0.6000 & 0.3333 & 0.0667 & 0.8667 \\
\hline Office & 0.2000 & 0.7333 & 0.8667 & 0.7333 & 0.4667 & 0.7333 & 0.0667 & 0.7333 \\
\hline Parking & 0.0667 & 0.8667 & 0.7333 & 0.4667 & 0.3333 & 0.7333 & 0.3333 & 1.0000 \\
\hline Radio & 0.0667 & 1.0000 & 0.7333 & 0.0667 & 0.7333 & 0.3333 & 0.3333 & 1.0000 \\
\hline Square & 0.0667 & 0.7333 & 0.8667 & 0.2000 & 0.7333 & 0.4667 & 0.0667 & 1.0000 \\
\hline Statue & 0.0667 & 0.8667 & 0.7333 & 0.4667 & 0.0667 & 0.7333 & 0.3333 & 0.8667 \\
\hline \multirow[t]{2}{*}{ Average } & 0.0959 & 0.7275 & 0.7078 & 0.2388 & 0.4122 & 0.5350 & 0.2591 & 0.8901 \\
\hline & \multicolumn{8}{|c|}{ Spearman Rank-Order Correlation Coefficient (SRCC) } \\
\hline Image Set & NMI [11] & EW-SSIM [16] & EIP [12] & SW-SSIM [17] & ES [18] & MEP [13] & SWCP [27] & FQI \\
\hline BelguimHouse & 0.0286 & 0.8857 & 1.0000 & 0.7714 & 0.1429 & 0.9429 & 0.0857 & 0.9429 \\
\hline Boat & 0.0857 & 0.6000 & 0.6000 & 0.2571 & 0.0857 & 0.1429 & 0.6571 & 0.9429 \\
\hline Cafa & 0.3143 & 0.9429 & 0.8857 & 0.1429 & 0.5429 & 0.7143 & 0.0857 & 0.9429 \\
\hline Church & 0.2000 & 0.8857 & 0.6571 & 0.0857 & 0.7714 & 0.5429 & 0.2571 & 0.9429 \\
\hline Foyer & 0.0857 & 0.9429 & 0.6571 & 0.0286 & 0.7714 & 0.7714 & 0.6000 & 0.9429 \\
\hline Garden & 0.2609 & 0.8986 & 0.8117 & 0.1739 & 0.9856 & 0.4638 & 0.5218 & 0.9856 \\
\hline House & 0.0579 & 0.7827 & 0.6377 & 0.2319 & 0.4058 & 0.4638 & 0.5508 & 0.9276 \\
\hline Lamp & 0.1429 & 0.7714 & 0.7714 & 0.0286 & 0.0857 & 0.6571 & 0.1429 & 0.9429 \\
\hline MAC & 0.0286 & 0.4286 & 0.8857 & 0.0857 & 0.7714 & 0.4857 & 0.0857 & 0.9429 \\
\hline Office & 143 & 86 & 0.9429 & 0.8286 & 0.6000 & 0.8286 & 0.0286 & 0.8286 \\
\hline Parking & 0.0286 & 0.9429 & 0.8286 & 0.6000 & 0.4857 & 0.8286 & 0.4857 & 1.0000 \\
\hline Radio & 0.0286 & 1.0000 & 0.8286 & 0.0286 & 0.8857 & 0.4857 & 0.4857 & 1.0000 \\
\hline Square & 0.0857 & 0.8286 & 0.9429 & 0.2571 & 0.8857 & 0.6000 & 0.0286 & 1.0000 \\
\hline Statue & 0.0286 & 0.9429 & 0.8286 & 0.6000 & 0.0857 & 0.8286 & 0.4857 & 0.9429 \\
\hline \multirow[t]{2}{*}{ Average } & 0.1207 & 0.8344 & 0.8056 & 0.2943 & 0.5361 & 0.6254 & 0.3215 & 0.9489 \\
\hline & \multicolumn{8}{|c|}{ Pearson Linear Correlation Coefficient (PLCC) } \\
\hline Image Set & NMI [11] & EW-SSIM [16] & EIP [12] & SW-SSIM [17] & ES [18] & MEP [13] & SWCP [27] & FQI \\
\hline BelguimHouse & -0.0643 & 0.9769 & 0.9919 & 0.5805 & 0.1328 & 0.7881 & 0.3556 & 0.9533 \\
\hline Boat & 0.0466 & 0.8283 & 0.6211 & -0.0703 & -0.1393 & 0.3976 & 0.5129 & 0.9444 \\
\hline Cafa & 0.3212 & 0.9736 & 0.9349 & 0.1998 & -0.2387 & 0.7449 & 0.3235 & 0.9621 \\
\hline Church & 0.1723 & 0.9169 & 0.7847 & 0.1237 & -0.7722 & 0.3885 & 0.6750 & 0.9276 \\
\hline Foyer & 0.2276 & 0.9217 & 0.8416 & 0.2549 & -0.5965 & 0.5288 & 0.7414 & 0.9341 \\
\hline Garden & 0.4392 & 0.7634 & 0.8795 & 0.3339 & -0.9092 & 0.4846 & 0.6603 & 0.9460 \\
\hline House & 0.2109 & 0.8974 & 0.8020 & 0.3808 & -0.5157 & 0.5656 & 0.7589 & 0.9611 \\
\hline Lamp & 0.0849 & 0.9555 & 0.7867 & 0.0185 & -0.0146 & 0.5564 & 0.3146 & 0.9820 \\
\hline MAC & 0.1457 & 0.8153 & 0.8875 & 0.0062 & -0.9279 & 0.5607 & 0.3769 & 0.9243 \\
\hline Office & 0.2067 & 0.9426 & 0.9464 & 0.7235 & -0.3555 & 0.6839 & 0.4607 & 0.9576 \\
\hline Parking & 0.1071 & 0.8872 & 0.9069 & 0.7744 & -0.1666 & 0.6783 & 0.7494 & 0.9765 \\
\hline Radio & 0.1747 & 0.9697 & 0.9702 & 0.4255 & -0.9278 & 0.7007 & 0.6127 & 0.9611 \\
\hline Square & 0.2927 & 0.9445 & 0.9794 & 0.2749 & -0.8739 & 0.6637 & 0.3302 & 0.9821 \\
\hline Statue & 0.0093 & 0.9511 & 0.9623 & 0.7740 & -0.0957 & 0.6681 & 0.7049 & 0.9894 \\
\hline Average & 0.1696 & 0.9103 & 0.8782 & 0.3429 & -0.4572 & 0.6007 & 0.5412 & 0.9573 \\
\hline
\end{tabular}

TABLE V

List of Algorithm Parameters Used in Fusion Quality Measures

\begin{tabular}{|l|l|}
\hline Fusion Quality Assessment Algorithm & Parameters \\
\hline Normalized mutual information (NMI) & no tuning parameter \\
Edge dependent weighted SSIM (EW-SSIM) & $\mathrm{C} 1$ and C2 are the default SSIM values set to $C_{1}=\left(K_{1} * L\right)^{2}$ and \\
& $C_{2}=\left(K_{2} * L\right)^{2}$ Where $K_{1}=0.01, K_{2}=0.03$ and $L=255$ \\
& $\alpha=1$ Window size to compute variance is $8 \times 8$ \\
Edge information preservation (EIP) & $\Gamma_{g}=\Gamma=1, k_{g}=10, k_{\alpha}=20, \sigma_{g}=0.5, \sigma_{\alpha}=0.75, L=1$ \\
Selective weighted SSIM (SW-SSIM) & Same default SSIM $C_{1}$, and $C_{2}, C_{3}=C_{2} / 2$, and sliding window \\
& $7 \times 7$ \\
Edge strength (ES) & $\alpha=5$ and local window size: $16 \times 16$ pixel \\
Multi-scale edge preservation (MEP) & $\alpha=1 / 3, \alpha_{2}=2 / 3, N=2$ \\
Saliency weighted contrast preservation (SWCP) & $k=h=1, p=3, q=2, Z=0.0001$ \\
\hline
\end{tabular}

be because the complication in the depth information in the real scene, which degrades the performance of the fusion algorithms and cast more challenges to fusion quality assessment measures as well.
The main computational cost of the proposed FQI algorithm is in the computation of LPC sharpness map, where an image is decomposed using $L$-scale $M$-orientation filtering with a total of one forward and $L M$ inverse $2 \mathrm{D}$ fast Fourier 

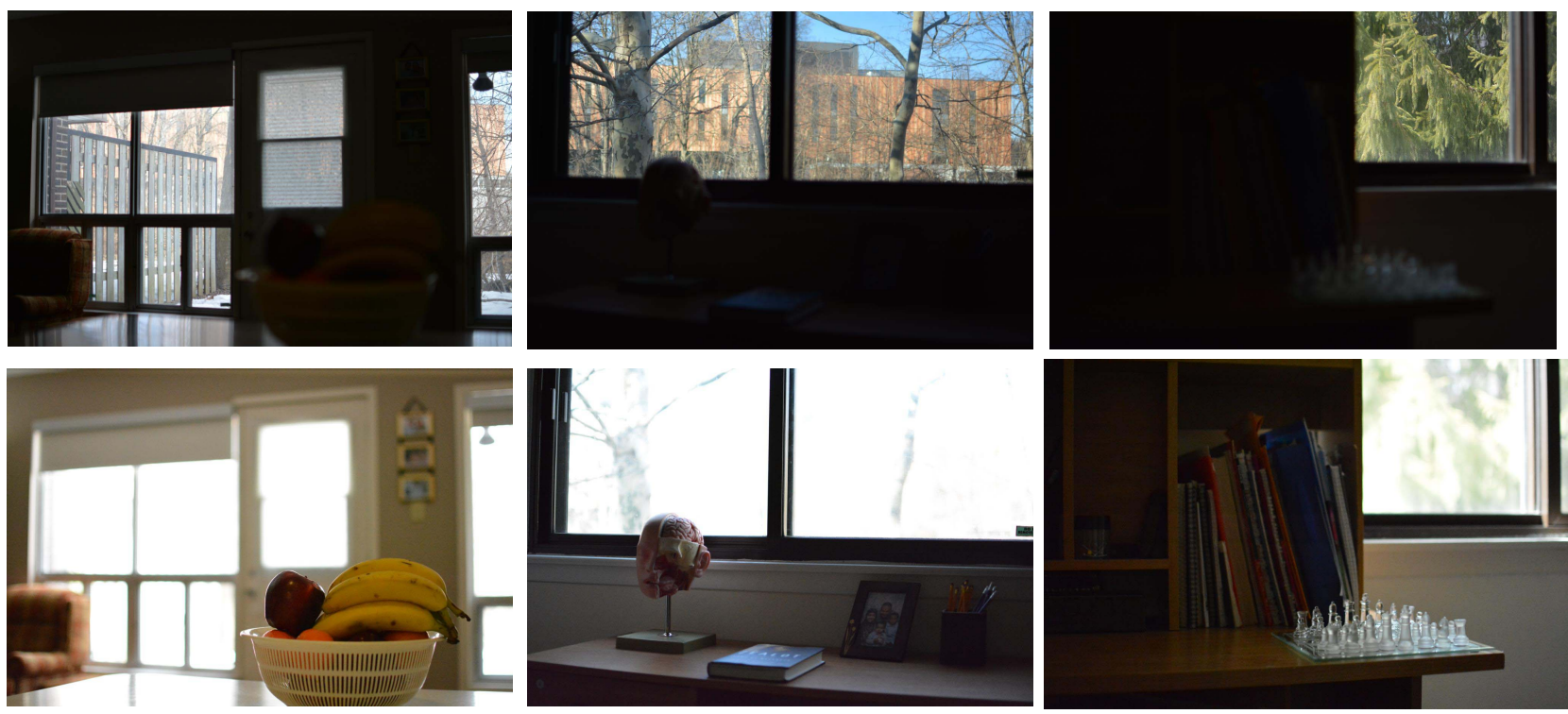

Fig. 7. Sample real multi-exposure multi-focus images captured using Nikon D5200 with AF-S DX NIKKOR 35mm f/1.8G lens.

TABLE VI

Statistical SignificAnCE ANALysis BAsED ON QUALITY PREDICTION RESIDUALS

\begin{tabular}{|c|cccccccc|}
\hline & NMI & EW-SSIM & EIP & SW-SSIM & ES & MEP & SWCP & FQI \\
\hline NMI & - & 0 & 0 & - & - & - & - & 0 \\
EW-SSIM & 1 & - & - & 1 & 1 & 1 & 1 & - \\
EIP & 1 & - & - & 1 & 1 & 1 & 1 & 0 \\
SW-SSIM & - & 0 & 0 & - & - & - & - & 0 \\
ES & - & 0 & 0 & - & - & - & - & 0 \\
MEP & - & 0 & 0 & - & - & - & - & 0 \\
SWCP & - & 0 & 0 & - & - & - & - & 0 \\
FQI & 1 & - & 1 & 1 & 1 & 1 & 1 & - \\
\hline
\end{tabular}

transforms (2D-FFTs). All other computations are linear with respect to the number of pixels $N$ in the image. Therefore, the computational complexity of the FQI is determined by the complexity of 2D-FFT and is thus on the order of $O(N \log N)$. When FQI is calculated for an image with $1120 \times 1120$ resolution using a computer configured with Intel Core i7 CPU at 2.40 GHz, 8GB RAM, Windows 7 64-bit, and unoptimized MATLAB code, the overall computation time is about 20 seconds. This gives a rough estimate of the current speed, and we expect that further algorithm, software and hardware optimizations could significantly improve the speed. Given its competitive quality prediction performance and reasonably fast speed, we believe that FQI has good potentials in real-world applications.

\section{Application: Parameter Tuning in IMAGE FUSION ALGORITHMS}

The application scope of objective image fusion quality measures is not limited to the validation and comparison of image fusion algorithms only. The area that has even greater potentials is to use the fusion quality models in the design and optimization of novel image fusion algorithms, which exhibit substantial variabilities in a broad range of applications in remote sensing, medical imaging, and high dynamic range imaging. Here we demonstrate this by applying the proposed FQI model in automatic parameter tuning of an image fusion algorithm, where the quality of the resulting fused image is highly sensitive to the image content and the parameters being tuned, making it difficult to pick the right parameters without human interactions.

In this work, we developed a wavelet domain spatially adaptive fusion algorithm. Given two input images $A$ and $B$, we first decompose the images using a discrete wavelet transform. This results in an approximation subband (low-low frequency band) and a series of detail subbands. Two different approaches are applied to fuse the coefficients in the detail and approximation subbands, respectively. In the detail subbands, for a pair of coefficients $c_{A}$ and $c_{B}$ at the same spatial location in the same wavelet subband of the two images, we first compute the standard deviations of their neighboring wavelet coefficients, denoted by $\sigma_{A}$ and $\sigma_{B}$, respectively. Presumably, the coefficient associated with larger value of local standard deviation contains more structural information and is of higher importance. To quantify their relative importance, we compute

$$
\lambda=\frac{\sigma_{A}}{\sigma_{A}+\sigma_{B}} .
$$

We can then create a fused coefficient $c_{F}$ by a weighted sum of $c_{A}$ and $c_{B}$ based on their importance:

$$
c_{F}=w_{A} c_{A}+\left(1-w_{A}\right) c_{B},
$$

where the weighting factor $w_{A}$ is bounded between 0 and 1 , and is determined by $\lambda$ using

$$
w_{A}= \begin{cases}0 & \text { if } \lambda<\alpha \\ \frac{\lambda-\alpha}{1-2 \alpha} & \text { if } \alpha \leq \lambda \leq(1-\alpha), \\ 1 & \text { if }(1-\alpha)<\lambda\end{cases}
$$


TABLE VII

SRCC Performance Evaluation of Image Fusion Quality Models Using Multi-Exposure Multi-Focus Images CAPtuREd in the ReAL ScEnes

\begin{tabular}{|c|c|c|c|c|c|c|c|c|}
\hline & \multicolumn{8}{|c|}{ Spearman Rank-Order Correlation Coefficient (SRCC) } \\
\hline Image Set & NMI [11] & EW-SSIM [16] & EIP [12] & SW-SSIM [17] & ES [18] & MEP [13] & SWCP [27] & FQI \\
\hline 1 & 0.5429 & 0.6000 & 0.2571 & 0.1429 & 0.6571 & 0.0857 & 0.0857 & 0.4857 \\
\hline 2 & 0.5429 & 1.0000 & 0.8857 & 0.8857 & 0.1429 & 0.6000 & 0.3143 & 0.9429 \\
\hline 4 & 0.3714 & 0.0857 & 0.6000 & 0.0286 & 0.7714 & 0.4286 & 0.1429 & 0.7714 \\
\hline 5 & 0.0000 & 0.7537 & 0.3769 & 0.4638 & 0.2319 & 0.2319 & 0.1160 & 0.7537 \\
\hline 6 & 0.1429 & 1.0000 & 0.9429 & 0.9429 & 0.4286 & 0.6000 & 0.0286 & 0.6000 \\
\hline 8 & 0.0304 & 0.8197 & 0.8197 & 0.6983 & 0.2732 & 0.5768 & 0.0911 & 0.8197 \\
\hline Average & 0.2576 & 0.6662 & 0.6621 & 0.5039 & 0.4171 & 0.4526 & 0.1367 & 0.7268 \\
\hline
\end{tabular}

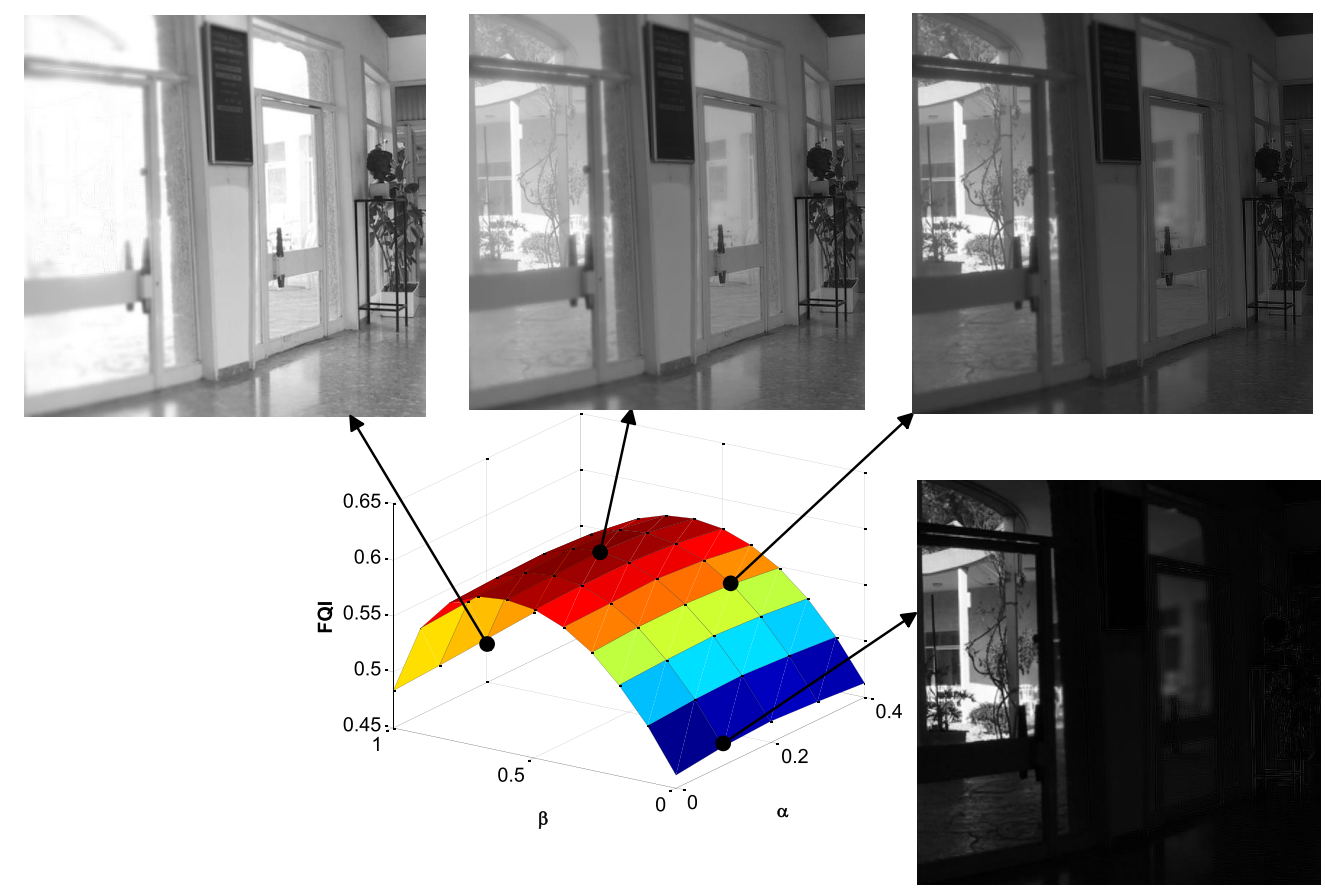

Fig. 8. FQI as a function of parameters $\alpha$ and $\beta$ in the wavelet domain adaptive fusion algorithm. Sample fused images are given for different selections of $\alpha$ and $\beta$ values.

where $\alpha$ is a parameter that can be adjusted within the range of $[0,0.5]$, producing different fusing results. In the approximation subband, every pair of coefficients $c_{A}$ and $c_{B}$ are fused to a new coefficient $c_{F}$ by a weighting factor $\beta$ :

$$
c_{F}=\beta c_{A}+(1-\beta) c_{B},
$$

where $\beta$ is another parameter that could affect the final outcome of the fusion algorithm. Finally, when all fused coefficients in all approximation and detail subbands are created using the above fusion methods, an inverse wavelet transform is applied to produce the final fused image $F$. A closely related pattern selection fusion approach can be found in [38], where a gradient-of-Gaussian pyramid was used to construct a pyramid transform for each source image and the fusion step is guided by two measures: a match measure based on normalized correlation between the source pyramids and a salience measure based on local energy. A weighted average combination rule is then used to construct the composite fused pyramid. It was demonstrated that this method is effective for diverse fusion applications of multi-sensor, multi-exposure, and multi-focus images.

In our experiment, we find that the above two-parameter ( $\alpha$ and $\beta$ ) fusion algorithm could create fused images with drastically different quality. Without human interaction, it becomes extremely difficult to find the best parameters, which largely depend on the image content. A quality assessment measure such as FQI is highly desirable to replace the role of humans, especially when the volume of images to be processed is large. Examples are given in Figs. 8 and 9, which plot FQI as a function of $\alpha$ and $\beta$ for two sets of source images, together with sample images corresponding to different selections of $\alpha$ and $\beta$ values. There are several observations from these figures. First, the quality of the fused images varies significantly with $\alpha$ and $\beta$. Second, the substantial difference in the 2D functions shown in Figs. 8 and 9 suggests that the best values of $\alpha$ and $\beta$ are sensitive to image content. Third, in both figures, the peak of the FQI function 


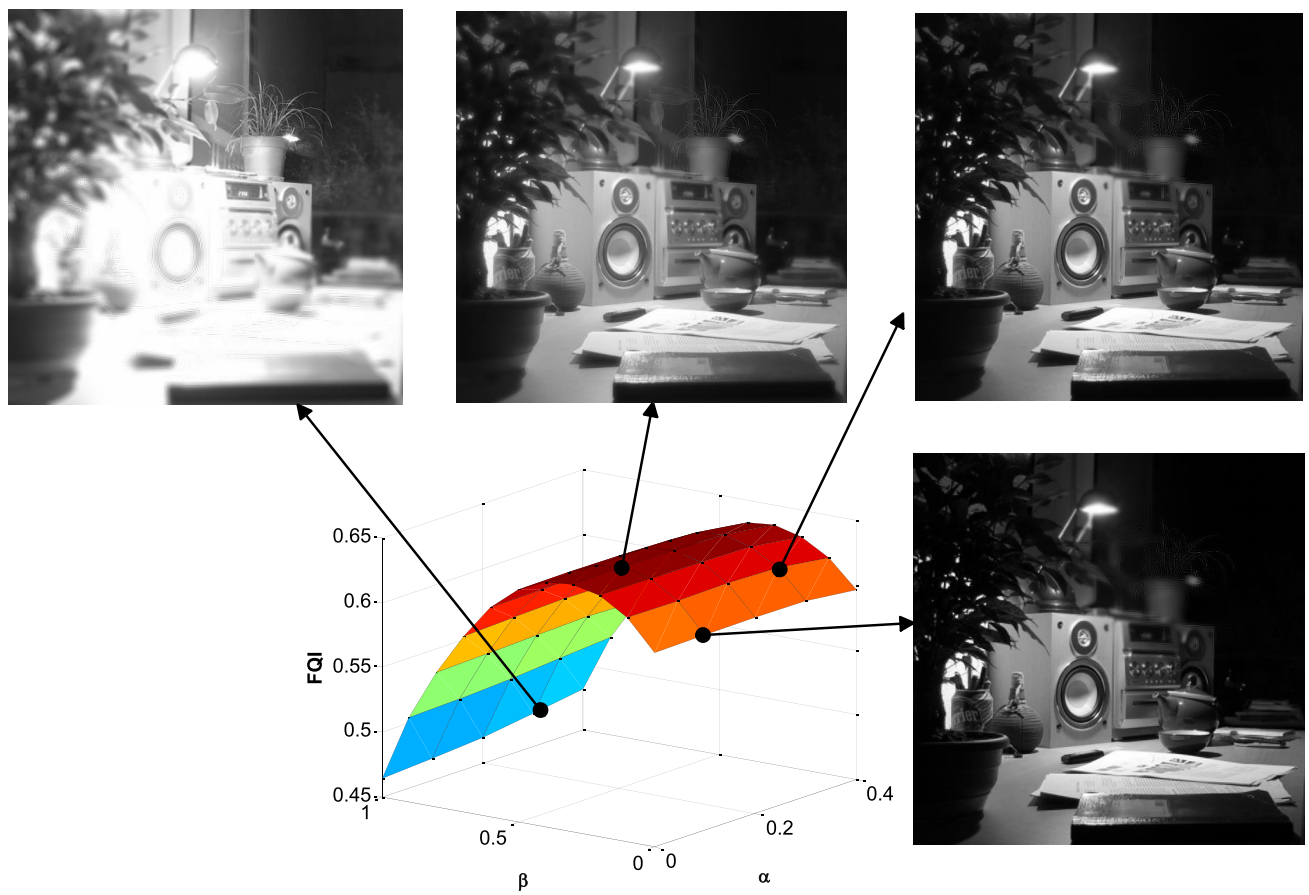

Fig. 9. FQI as a function of parameters $\alpha$ and $\beta$ in the wavelet domain adaptive fusion algorithm. Sample fused images are given for different selections of $\alpha$ and $\beta$ values.

corresponds to the images with the highest quality, indicating that FQI provides a useful index to automatically pick the best parameters for the fusion algorithm.

\section{CONClusions}

The major contributions of this work are as follows. Firstly, we propose an FQI algorithm for objective quality assessment of multi-exposure multi-focus image fusion by incorporating contrast preservation, sharpness, and structural preservation as the key factors; Secondly, we create a multi-exposure multifocus image fusion database, based on which a subjective quality assessment experiment is conducted. The database and the associated subjective test scores provide a useful test bed, probably the first of its kind, for image fusion quality assessment research. Based on KRCC, SRCC, PLCC, similarity dendrogram, and statistical significance analysis carried out on the database, the proposed FQI method outperforms seven stateof-the-art image fusion quality measures. Third, we captured real multi-exposure multi-focus image data set and carried out a subjective test on the real images. The real data set and the subjective scores were used to verify the performance of the proposed FQI method, which outperforms existing measures. Finally, we demonstrate the potential applications of FQI by employing it for parameter tunings of image fusion algorithms, where we show FQI is a powerful tool in selecting the optimal parameters that are highly sensitive to image content.

In general, the research area of image fusion quality assessment is still at a premature stage and the future work can be extended in many directions. The case of 2 input images tested in the current work often corresponds to the worst case scenario for image fusion algorithms and provides a strong test that differentiates both image fusion algorithms and fusion quality assessment models. If a fusion quality assessment algorithm does not work properly in 2-input image cases, then one would not expect it to work well in multiple input image cases. Conducting subjective tests on more than 2-input images and using more fusion algorithms would be natural extensions of the current work. Further extensions also include assessing the quality of images created by combing multiple images that are not perfectly aligned with each other; images fused from hyperspectral images in remote sensing applications; and images generated by merging different imaging modalities in medical imaging applications.

\section{REFERENCES}

[1] Z. Zhang and R. S. Blum, "A categorization of multiscaledecomposition-based image fusion schemes with a performance study for a digital camera application," Proc. IEEE, vol. 87, no. 8, pp. 1315-1326, Aug. 1999.

[2] R. S. Blum, Z. Xue, and Z. Zhang, "An overview of image fusion," in Multi-Sensor Image Fusion and Its Applications. New York, NY, USA: Marcel Dekker, 2006.

[3] Z. Wang, D. Ziou, C. Armenakis, D. Li, and Q. Li, "A comparative analysis of image fusion methods," IEEE Trans. Geosci. Remote Sens., vol. 43, no. 6, pp. 1391-1402, Jun. 2005.

[4] S. T. C. Wong, R. C. Knowlton, R. A. Hawkins, and K. D. Laxer, "Multimodal image fusion for noninvasive epilepsy surgery planning," IEEE Comput. Graph. Appl., vol. 16, no. 1, pp. 30-38, Jan. 1996.

[5] A. A. Goshtasby, "Fusion of multi-exposure images," Image Vis. Comput., vol. 23, no. 6, pp. 611-618, 2005.

[6] S. Li, J. T.-Y. Kwok, I. W.-H. Tsang, and Y. Wang, "Fusing images with different focuses using support vector machines," IEEE Trans. Neural Netw., vol. 15, no. 6, pp. 1555-1561, Nov. 2004.

[7] B. Yang and S. Li, "Multifocus image fusion and restoration with sparse representation," IEEE Trans. Instrum. Meas., vol. 59, no. 4, pp. 884-892, Apr. 2010.

[8] G. Piella, "A general framework for multiresolution image fusion: From pixels to regions," Inf. Fusion, vol. 4, no. 4, pp. 259-280, 2003. 
[9] H. Li, B. S. Manjunath, and S. K. Mitra, "Multisensor image fusion using the wavelet transform," Graph. Models Image Process., vol. 57, no. 3 , pp. $235-245,1995$.

[10] O. Rockinger, "Image sequence fusion using a shift-invariant wavelet transform," in Proc. Int. Conf. Image Process., vol. 3. Oct. 1997, pp. 288-291.

[11] G. Qu, D. Zhang, and P. Yan, "Information measure for performance of image fusion," Electron. Lett., vol. 38, no. 7, pp. 313-315, 2002.

[12] C. S. Xydeas and V. Petrovic, "Objective image fusion performance measure," Electron. Lett., vol. 36, no. 4, pp. 308-309, 2000.

[13] P.-W. Wang and B. Liu, "A novel image fusion metric based on multiscale analysis," in Proc. 9th Int. Conf. Signal Process., Oct. 2008, pp. $965-968$.

[14] Z. Wang, A. C. Bovik, H. R. Sheikh, and E. P. Simoncelli, "Image quality assessment: From error visibility to structural similarity," IEEE Trans. Image Process., vol. 13, no. 4, pp. 600-612, Apr. 2004.

[15] Z. Wang and A. C. Bovik, "A universal image quality index," IEEE Signal Process. Lett., vol. 9, no. 3, pp. 81-84, Mar. 2002.

[16] G. Piella and H. Heijmans, "A new quality metric for image fusion," in Proc. IEEE Int. Conf. Image Process., vol. 3. Sep. 2003, pp. III-173-III-176.

[17] C. Yang, J.-Q. Zhang, X.-R. Wang, and X. Liu, "A novel similarity based quality metric for image fusion," Inf. Fusion, vol. 9, no. 2, pp. 156-160, 2008.

[18] H. Chen and P. K. Varshney, "A human perception inspired quality metric for image fusion based on regional information," Inf. Fusion, vol. 8, no. 2, pp. 193-207, 2007.

[19] R. Ferzli and L. J. Karam, "A no-reference objective image sharpness metric based on the notion of just noticeable blur (JNB)," IEEE Trans. Image Process., vol. 18, no. 4, pp. 717-728, Apr. 2009.

[20] C. T. Vu, T. D. Phan, and D. M. Chandler, "S 3 : A spectral and spatial measure of local perceived sharpness in natural images," IEEE Trans. Image Process., vol. 21, no. 3, pp. 934-945, Mar. 2012.

[21] R. Hassen, Z. Wang, and M. Salama, "Multifocus image fusion using local phase coherence measurement," in Proc. 6th Int. Conf. Image Anal. Recognit., 2009, pp. 54-63.

[22] R. Hassen, Z. Wang, and M. M. A. Salama, "Image sharpness assessment based on local phase coherence," IEEE Trans. Image Process., vol. 22, no. 7, pp. 2798-2810, Jul. 2013.

[23] A. Toet and E. M. Franken, "Perceptual evaluation of different image fusion schemes," Displays, vol. 24, no. 1, pp. 25-37, 2003.

[24] Y. Rubner, J. Puzicha, C. Tomasi, and J. M. Buhmann, "Empirical evaluation of dissimilarity measures for color and texture," Comput. Vis. Image Understand., vol. 84, pp. 25-43, 2001.

[25] V. Petrović, "Subjective tests for image fusion evaluation and objective metric validation," Inf. Fusion, vol. 8, no. 2, pp. 208-216, 2007.

[26] P. Ledda, A. Chalmers, T. Troscianko, and H. Seetzen, "Evaluation of tone mapping operators using a high dynamic range display," $A C M$ Trans. Graph., vol. 24, no. 3, pp. 640-648, 2005.

[27] Y. Chen and R. S. Blum, "A new automated quality assessment algorithm for image fusion," Image Vis. Comput., vol. 27, no. 10, pp. 1421-1432, 2009.

[28] F. Lukas and Z. L. Budrikis, "Picture quality prediction based on a visual model," IEEE Trans. Commun., vol. 30, no. 7, pp. 1679-1692, Jul. 1982.

[29] CCIR, "Method of the subjective assessment of the quality of television pictures," in Proc. 13th Plenary Assembly, Rec., vol. 11. 1974, pp. 65-68.

[30] Z. Wang and A. C. Bovik, "Embedded foveation image coding," IEEE Trans. Image Process., vol. 10, no. 10, pp. 1397-1410, Oct. 2001.

[31] R. Shen, I. Cheng, J. Shi, and A. Basu, "Generalized random walks for fusion of multi-exposure images," IEEE Trans. Image Process., vol. 20, no. 12, pp. 3634-3646, Dec. 2011.

[32] O. Rockinger. Image Fusion Toolbox. [Online]. Available: http://www. metapix.de/toolbox.htm

[33] L. L. Thurstone, "A law of comparative judgment," Psychol. Rev., vol. 34, no. 4, pp. 273-286, 1927.

[34] R. D. Luce, "Thurstone and sensory scaling: Then and now," Psychol. Rev., vol. 101, no. 2, pp. 271-277, Apr. 1994.

[35] R. R. Sokal and C. D. Michener, A Statistical Method for Evaluating Systematic Relationships. Lawrence, KS, USA: Univ. Kansas, 1958.
[36] F. Murtagh, "Complexities of hierarchic clustering algorithms: State of the art," Comput. Statist. Quart., vol. 1, no. 2, pp. 101-113, 1984.

[37] H. R. Sheikh, M. F. Sabir, and A. C. Bovik, "A statistical evaluation of recent full reference image quality assessment algorithms," IEEE Trans. Image Process., vol. 15, no. 11, pp. 3440-3451, Nov. 2006.

[38] P. J. Burt and R. J. Kolczynski, "Enhanced image capture through fusion," in Proc. 4th IEEE Int. Conf. Comput. Vis., May 1993, pp. 173-182.

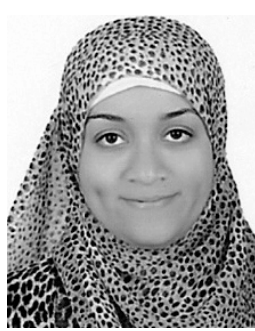

Rania Hassen received the Ph.D. degree in electrical and computer engineering from the University of Waterloo, Waterloo, Canada, in 2013. She is currently an Assistant Professor with the Department of Computer Science, Assiut University, Egypt. Her research interests include image processing, image quality assessment, medical imaging, and biomedical image processing. She received the Ontario Graduate Student Science and Technology Scholarship, and the Doctoral Award for Women in Engineering during her post-graduate study at the

University of Waterloo.

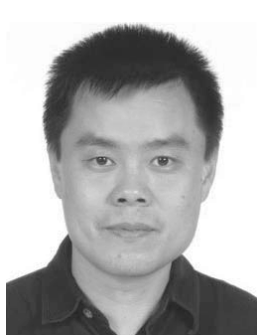

Zhou Wang (S'99-M'02-SM'12-F'14) received the $\mathrm{Ph} . \mathrm{D}$. degree in electrical and computer engineering from The University of Texas at Austin in 2001. He is currently an Associate Professor with the Department of Electrical and Computer Engineering, University of Waterloo, Canada. He has authored over 100 publications in his research fields with over 24000 citations (a Google Scholar). His research interests include image processing, coding, and quality assessment; computational vision and pattern analysis; multimedia communications; and biomedical signal processing. He was a member of the IEEE Multimedia Signal Processing Technical Committee (2013-2015). He served as an Associate Editor of the IEEE TRANSACTIONS ON IMAgE PROCESSING (2009-2014), Pattern Recognition (2006-present), and the IEEE Signal Processing LETTERS (2006-2010), and a Guest Editor of the IEEE JOURNAL OF Selected Topics in Signal Processing (2013-2014 and 2007-2009), the EURASIP Journal of Image and Video Processing (2009-2010), and Signal, Image and Video Processing (2011-2013). He was a recipient of the 2014 NSERC E. W. R. Steacie Memorial Fellowship Award, the 2013 IEEE Signal Processing Best Magazine Paper Award, the 2009 IEEE Signal Processing Society Best Paper Award, the 2009 Ontario Early Researcher Award, and the ICIP 2008 IBM Best Student Paper Award (as a senior author).

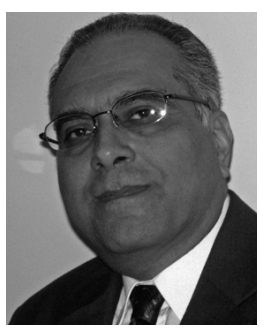

Magdy M. A. Salama received the B.Sc. and M.S. degrees from Cairo University, Cairo, Egypt, in 1971 and 1973, respectively, and the Ph.D. degree from the University of Waterloo, Waterloo, ON, Canada, in 1977, all in electrical engineering. He has consulted widely with government agencies and the electrical authority. He is currently a Professor with the Electrical and Computer Engineering Department, University of Waterloo. He is a Registered Professional Engineer in the Province of Ontario. His research interests include the operation and control of electric distribution systems and biomedical engineering applications. 